\title{
Estudio de la esquizofrenia comórbida con la adicción alcohólica
}

\author{
Vlasta Solter MD, PhD \\ Vlatko Thaller MD, PhD \\ Ante Bagarić MD \\ Dalibor Karlović MD, MS \\ Danijel Crnković MD \\ Jelena Potkonjak MD \\ University Department of Psychiatry, \\ University Hospital "Sestre milosrdnice", \\ Zagreb \\ CROATIA
}

\begin{abstract}
RESUMEN - El objetivo de este estudio fue investigar: la prevalencia de dependencia alcohólica actual, la anamnesis familiar de la adicción alcohólica, las características sociales y demográficas, el número de hospitalizaciones y el grado de cumplimiento con la psicofarmacoterapia, como predictores de mala evolución de la esquizofrenia comórbida con la adicción alcohólica. Nuestros resultados muestran que en los varones con esquizofrenia las tasas de prevalencia de adicción alcohólica comórbida son más altas que en mujeres.

La comorbilidad de la adicción alcohólica y la esquizofrenia en hombres se relaciona con mayores tasas de divorcio y con la edad. En mujeres, la comorbilidad adicción alcohólica y esquizofrenia se relaciona con un nivel bajo de educación, una tasa de solteras más alta y con seguro de enfermedad familiar. Hay una fuerte evidencia de adicción alcohólica en familiares de primer grado de esquizofrénicos con adicción alcohólica comórbida, tanto hombres como mujeres. La adicción alcohólica comórbida con esquizofrenia en hombres y mujeres se relaciona con una mala cumplimentación del tratamiento psicofarmacológico y con tasas más altas de hospitalización, comparada con esquizofrenia sola.
\end{abstract}

\section{Introducción}

La esquizofrenia es sinónimo de trastorno mental grave con una incidencia relativamente alta, un curso crónico, y un cuadro clínico abigarrado (Brown y White 1992, Hafner y Heiden 1997).
Los pacientes con esquizofrenia a menudo sufren daño social, cognitivo y biológico (Hafner y Heiden 1997, Bleuler 1911). Consecuentemente, el pronóstico de los pacientes esquizofrénicos puede ser excepcionalmente malo, especialmente si la enfermedad es comórbida con algún otro trastorno psiquiátri- 
co (Fenton y McGlasham 1991). La prevalencia más alta de éstos es la adicción alcohólica, seguida de los trastornos de personalidad y de distintos trastornos afectivos (Penick et al. 1994). La esquizofrenia, en comorbilidad con la adicción alcohólica, puede tener un curso extraordinariamente desfavorable (Guebaly y Hodkins 1992, Dixon 1999). Esto es debido al hecho de que la adicción alcohólica lleva a una reducción de las funciones sociales y cognitivas, causa daño somático y, finalmente, puede causar cuadros clínicos psicóticos muy similares a los de la esquizofrenia misma (Nordsy et al. 1994, Thaller 1999, Thaller et al. 1996, Rubin 1993). Hay muchas causas para la adicción alcohólica: psicológicas, socioculturales, neuroquímicas y genéticas (Berman 1990, Lovinger et al. 1989, Cloninger et al. 1981).

Las últimas investigaciones han señalado que la incidencia de adicción alcohólica en esquizofrénicos es cuatro veces más alta que en población general (Helzer y Pryzbeck 1988). Los pacientes con formas esquizofrénicas paranóicas con alucinaciones desarrollan adicción alcohólica más frecuentemente que los demás tipos de esquizofrenia (Batel 2000). Sus razones para tomar alcohol son un tipo de proceso de autotratamiento con el propósito principal de eliminar los efectos secundarios (Cantor et al. 2001).

Los factores sociales, la aceptación de las bebidas alcohólicas en algunas culturas, y también la heredabilidad, causan adicción alcohólica comórbida con esquizofrenia. A la vista de estos hechos, el objetivo de nuestra investigación es definir la prevalencia de adicción alcohólica en pacientes esquizofrénicos. El segundo propósito es examinar los antecedentes familiares, en relación con la heredabilidad psiquiátrica, y analizar los cuadros clínicos sociales y familiares en pacientes esquizofrénicos hospitalizados con y sin adicción alcohólica. El tercer objetivo es establecer si la esquizofrenia comórbida con adicción alcohólica tiene mal pronóstico. Investigaremos esto usando la información referente al número de hospitalizaciones durante un año, y si el paciente está cumpliendo el tratamiento o no.

\section{Material y métodos}

\section{Muestra}

De los pacientes $(\mathrm{N}=15.061)$ que fueron tratados en el Departamento de Psiquiatría de la Clínica Universitaria "Sestre Milosrdnice" entre el 1 de enero de 1995 y el 1 de enero de 2000, seleccionamos aquéllos a los que se les diagnosticó esquizofrenia, o esquizofrenia comórbida con adicción alcohólica. Los pacientes que fueron diagnosticados de esquizofrenia comórbida con otra forma de trastorno psiquiátrico fueron excluídos del estudio. Usamos este procedimiento para obtener pacientes más homogéneos pero con una mejor diferenciación diagnóstica. Finalmente, obtuvimos 794 pacientes, hombres $(\mathrm{N}=430)$ y mujeres $(\mathrm{N}$ = 364). Todos ellos dieron su consentimiento para participar en el estudio. Los parámetros descriptivos para ambos géneros son presentados en la Tabla I.

La edad media \pm una desviación estándar de los hombres incluidos en el estudio fue de 37,46 $\pm 10,4$ años, y para las mujeres fue de 41,7 $\pm 12,7$ años. La mayoría tenía un nivel educativo de bachillerato $(57,7 \%$ hombres y 50,3\% mujeres). La mayoría de los hombres eran solteros (57\%), y la mayoría de las mujeres estaban casadas (42\%). La mayor parte de la muestra tenía un seguro de vida $(77,2 \%$ hombres y $59,9 \%$ mujeres). 
Tabla I

Características socio-demográficas de los pacientes esquizofrénicos estudiados $(\mathrm{N}=794)$

\begin{tabular}{|c|c|c|}
\hline & Hombres N (\%) & Mujeres N (\%) \\
\hline \multicolumn{3}{|l|}{ Años de edad } \\
\hline $21-30$ & $98(22,8)$ & $60(16,5)$ \\
\hline $31-40$ & $166(38,6)$ & $105(28,8)$ \\
\hline $41-50$ & $114(26,5)$ & $105(28,8)$ \\
\hline $51-60$ & $39(9,1)$ & $56(15,4)$ \\
\hline$>60$ & $13(3,0)$ & $38(10,5)$ \\
\hline \multicolumn{3}{|l|}{ Estado civil } \\
\hline Casado & $150(34,9 \%)$ & $153(42,0 \%)$ \\
\hline Soltero & $245(57,0 \%)$ & $128(35,2 \%)$ \\
\hline Divorciado & $32(7,4 \%)$ & $70(19,2 \%)$ \\
\hline Viudo & $3(0,7 \%)$ & $13(3,6 \%)$ \\
\hline \multicolumn{3}{|l|}{ Nivel educativo } \\
\hline Estudios primarios & $127(29,5 \%)$ & $135(37,1 \%)$ \\
\hline Bachillerato & $248(57,7 \%)$ & $183(50,3 \%)$ \\
\hline Formación Profesional & $22(5,1 \%)$ & $14(3,8 \%)$ \\
\hline Universidad & $33(7,7 \%)$ & $32(8,8 \%)$ \\
\hline \multicolumn{3}{|l|}{ Seguro de vida } \\
\hline Activo & $332(772 \%)$ & $218(59,9 \%)$ \\
\hline Familiar & $77(17,9 \%)$ & $124(34,1 \%)$ \\
\hline Sin seguro & $21(4,9 \%)$ & $22(6,0 \%)$ \\
\hline \multicolumn{3}{|l|}{ Lugar de residencia } \\
\hline Urbano & $375(87,2 \%)$ & $326(89,6 \%)$ \\
\hline Rural & $55(12,8 \%)$ & $38(10,4 \%)$ \\
\hline
\end{tabular}

Tres cuartas partes de la muestra eran de la ciudad (87,2\% hombres y $89,5 \%$ mujeres).

\section{Instrumentos}

1. La entrevista clínica estructurada usada para este estudio contiene información sobre el estatus socioeconómico y características demográficas de los pacientes (edad, sexo, educación, estado civil, número de hijos, tipo de seguro de vida, y lugar de residencia-urbana o rural).

2. Una entrevista clínica estructurada para los pacientes y sus familiares, incluyendo información sobre la herencia genética de enfermedades mentales (madre, padre, abuelo, abuela, tíos, etc.), e información sobre medicación, así como también número de hospitalizaciones.
3. Una entrevista clínica estructurada basada en los criterios DSM IV, con información crucial para el diagnóstico de esquizofrenia y de adicción alcohólica (American Psychiatric Association 1994).

4. El cuestionario CAGE fue empleado como instrumento de screening para problemas/adicción grave de alcohol (Ewing 1984, King 1986). Este cuestionario fue utilizado por su simplicidad y fiabilidad.

\section{Procedimiento}

La investigación se diseñó como un estudio transversal retrospectivo, ya descrito en publicaciones previas (Regier 1995). Los psiquiatras y los residentes de psiquiatría llevaron a cabo una entrevista clínica estructurada, e hicieron un diagnóstico basado en los criterios DSM IV para esquizofrenia. El 
diagnóstico final de esquizofrenia fue hecho sólo si ambos evaluadores habían llegado al diagnóstico. El acuerdo diagnóstico entre ambos evaluadores basado en la entrevista clínica estructurada fue del $97 \%$. Aceptamos el diagnóstico de "adicción alcohólica" sólo si el paciente reunía dos criterios: resultado indicativo en el cuestionario CAGE, y un número suficiente de síntomas mostrados en la entrevista clínica estructurada basada en los criterios DSM IV para adicción alcohólica. El acuerdo diagnóstico entre el cuestionario CAGE y la investigación médica referente a la adicción alcohólica fue del $98 \%$.

\section{Análisis estadístico}

Calculamos las tasas de prevalencia de comorbilidad de adicción alcohólica en esquizofrenia por separado para hombres y mujeres como se describe en publicaciones previas (Regier 1995). La comparación de la tasa de adicción alcohólica en hombres y mujeres esquizofrénicos se realizó calculando los valores chi cuadrado. Los sujetos esquizofrénicos, con y sin adicción alcohólica se compararon por edad mediante el test t de Student, y para el estado civil, nivel de educación, seguro de vida y lugar de residencia por medio del test de chi cuadrado.

La relación entre la psicopatología de los familiares y los sujetos esquizofrénicos fue analizada con el test de chi cuadrado.

Para analizar el papel de la adicción alcohólica comórbida con esquizofrenia en el número de hospitalizaciones por año y en el cumplimiento de la psicofarmacoterapia se hicieron cuatro grupos: hombres esquizofrénicos con y sin adicción alcohólica y mujeres esquizofrénicas con y sin adicción alcohólica. Estos grupos fueron comparados mediante el estudio del número de hospitali- zaciones por año o del rango de cumplimiento de la psicofarmacoterapia a través de un análisis de la varianza (ANOVA) y análisis Scheffe's post-hoc. Se utilizó el programa informático SPSS 8.0 para Windows en todos los análisis.

\section{Resultados}

La tasa de prevalencia de adicción alcohólica comórbida con esquizofrenia es más alta en hombres que en mujeres: $10,2 \%$ vs. $6,6 \%$, pero estadísticamente no significativa $\left(\mathrm{chi}^{2}=2,9 ; \mathrm{gl}=1 ; \mathrm{p}>0.1\right)$.

Hubo diferencias estadísticamente significativas por edad en los pacientes varones con esquizofrenia comórbida con adicción alcohólica comparados con los pacientes varones con esquizofrenia sola (media \pm $\mathrm{SD} ; 42,4 \pm 11,6$, vs. $36,9 \pm 10,1 ; \mathrm{t}=3,368$; $\mathrm{p}<0,001)$. No hubo diferencias por edad entre pacientes mujeres con esquizofrenia comórbida con adicción alcohólica y pacientes mujeres con esquizofrenia sola (media $\pm \mathrm{SD} ; 41,6 \pm 11,5$, vs. $41,7 \pm 12,8 ; \mathrm{t}$ $=0,021 ; \mathrm{p}=0,983)$.

En las mujeres, la esquizofrenia con adicción alcohólica comórbida se relaciona con bajo nivel de educación, viven en un área urbana y tienen seguro familiar de enfermedad. La esquizofrenia comórbida con adicción alcohólica en hombres se relaciona con una alta tasa de divorcio (Tabla II).

Analizando el papel de los factores hereditarios familiares en relación con la esquizofrenia con adicción alcohólica comórbida se ha visto que los pacientes, tanto mujeres como hombres, tienen familiares cercanos con adicción alcohólica así como también esquizofrenia (Tabla III). 
Tabla II

Comparación del grupo de pacientes esquizofrénicos con el grupo de pacientes con esquizofrenia comórbida con dependencia alcohólica, según estado civil, nivel educativo, seguro, entorno, y edad

\begin{tabular}{lcccl} 
& Esquizofrenia & $\begin{array}{c}\text { Esquizofrenia }+ \\
\text { dependencia alcohólica }\end{array}$ & $\begin{array}{c}\text { Chi-cuadrado } \\
(\mathrm{gl})\end{array}$ & $\mathrm{p}$ \\
\hline Hombres & $(\%, \mathrm{n}=386)$ & $(\%, \mathrm{n}=44)$ & & \\
$\quad$ Educación secundaria & 57,6 & 63,5 & $1,208(3)$ & 0,751 \\
Soltero & 6,0 & 20,0 & $14,032(3)$ & 0,00287 \\
Seguro activo & 76,5 & 84,1 & $1,484(3)$ & 0,476 \\
Área urbana & 87,8 & 81,9 & $1,277(1)$ & 0,2584 \\
\hline Mujeres & $(\%, \mathrm{n}=340)$ & $(\%, \mathrm{n}=24)$ & & \\
Educación secundaria & 36,2 & 50,1 & $15,861(3)$ & 0,00121 \\
Casada & 35,1 & 33,2 & $4,062(3)$ & 0,255 \\
Seguro familliar & 83,4 & 58,2 & $6,194(3)$ & 0,0452 \\
Área urbana & 88,8 & 100,0 & $2,995(1)$ & 0,08353 \\
\hline
\end{tabular}

Tabla III

Trastornos psiquiátricos familiares en pacientes esquizofrénicos con y sin dependencia alcohólica

\begin{tabular}{lccccc} 
& $\begin{array}{c}\text { Sin } \\
\text { Patología } \\
\%\end{array}$ & $\begin{array}{c}\text { Alcoholismo } \\
\text { Esquizofrenia }\end{array}$ & $\begin{array}{c}\text { Trastornos } \\
\text { Afectivos } \\
\%\end{array}$ & $\begin{array}{c}\text { Chi } \\
\text { cuadrado }\end{array}$ \\
\hline $\begin{array}{l}\text { Hombres }(\mathrm{n}=430) \\
\text { Esquizofrenia }\end{array}$ & 58,3 & 23,6 & 12,7 & 5,7 & $19,5^{\mathrm{a}}$ \\
$\begin{array}{l}\text { Esquizofrenia }+ \\
\text { Dependencia alcohólica }\end{array}$ & 28,8 & 52,3 & 14,2 & 4,5 & $18,9^{\mathrm{a}}$ \\
\hline $\begin{array}{l}\text { Mujeres (n = 364) } \\
\text { Esquizofrenia }\end{array}$ & 59,7 & 17,9 & 19,9 & 2,6 & \\
$\begin{array}{l}\text { Esquizofrenia }+ \\
\text { Dependencia alcohólica }\end{array}$ & 37,5 & 45,8 & 16,7 & 0,0 & \\
\hline
\end{tabular}

(a) $\mathrm{p}<0,001$.

Para analizar el papel de la adicción alcohólica comórbida con esquizofrenia en relación con el número de hospitalizaciones por año y con la cumplimentación de la psicofarmacoterapia, se compararon cuatro grupos (grupos con esquizofrenia y grupos con esquizofrenia comórbida con adicción alcohólica, ambos separadamente para hombres y mujeres) (Tablas IV y V). El número más alto de hospitalizaciones, y peor cumplimentación psicofarmacológica se halló en pacientes mujeres esquizofrénicas con adicción alcohólica comórbida, seguido de los pacientes hombres esquizofrénicos con adicción alcohólica comórbida. Los análisis Scheffe's post-hoc mostraron que no hay diferencias en el número de hospitalizaciones ni en cumplimentación terapéutica entre esquizofrénicos hombres y mujeres con adicción alcohólica comórbida, en comparación con pacientes de ambos géneros, con esquizofrenia sola. 
Tabla IV

Número de hospitalizaciones en relación con el diagnóstico de esquizofrenia y esquizofrenia comórbida con dependencia alcohólica, en hombres y mujeres

\begin{tabular}{lcc} 
Grupos & $\begin{array}{c}\text { Media de } \\
\text { hospitalizaciones }\end{array}$ & $\begin{array}{c}\text { Test de probabilidad de Scheffe, } \\
\text { diferencias entre grupos individuales }\end{array}$ \\
\hline $\begin{array}{l}\text { 1. Esquizofrenia, hombres } \\
\text { 2. Esquizofrenia + dependencia } \\
\text { alcohólica, hombres }\end{array}$ & $1,2 \pm 0,5$ & $\mathrm{p}(1,2)<0,001$ \\
$\begin{array}{l}\text { 3. Esquizofrenia, mujeres } \\
\text { 4. Esquizofrenia + dependencia } \\
\text { alcohólica, mujeres }\end{array}$ & $2,4 \pm 0,5$ & $\mathrm{p}(1,3)=0,06$ \\
$\mathrm{p}(1,4)<0,001$ \\
$\mathrm{p}(2,3)<0,001$ \\
$\mathrm{P}$
\end{tabular}

Análisis de la varianza, $\mathrm{F}=8,6 ; \mathrm{p}=0,003$.

Table V

Cumplimentación de la psicofarmacoterapia en relación con el diagnóstico de esquizofrenia y de esquizofrenia comórbida con dependencia alcohólica, en hombres y mujeres

\begin{tabular}{lcc} 
Grupos & $\begin{array}{c}\text { Media de } \\
\text { hospitalizaciones }\end{array}$ & $\begin{array}{c}\text { Test de probabilidad de Scheffe, } \\
\text { diferencias entre grupos individuales }\end{array}$ \\
\hline $\begin{array}{l}\text { 1. Esquizofrenia, hombres } \\
\text { 2. Esquizofrenia + dependencia } \\
\text { alcohólica, hombres }\end{array}$ & $0,1 \pm 0,3$ & $\mathrm{p}(1,2)<0,001$ \\
$\begin{array}{l}\text { 3. Esquizofrenia, mujeres } \\
\text { 4. Esquizofrenia + dependencia } \\
\text { alcohólica, mujeres }\end{array}$ & $0,8 \pm 0,4$ & $\mathrm{p}(1,3)=0,9$ \\
$\mathrm{p}(1,4)<0,001$ \\
$\mathrm{p}(2,3)<0,001$ \\
$\mathrm{p}(2,4)=0,9$ \\
$\mathrm{p}(3,4)<0,001$
\end{tabular}

Análisis de la varianza, $\mathrm{F}=0,1 ; \mathrm{p}=0,9$.

\section{Discusión}

No se ha llevado a cabo nunca en una población croata una investigación antropológica acerca de la comorbilidad de la esquizofrenia y la adicción alcohólica. Nuestro estudio además incluyó el análisis de los familiares de los pacientes.

En nuestra investigación, la prevalencia de adicción alcohólica en hombres y mujeres esquizofrénicos está en un rango del 3\% al 60\% (Penick et al. 1994, Guebaly y Hodkins 1992, Dixon 1999). Dicho rango tan amplio de prevalencia es el resultado de los diferentes tipos de investigación, y también de la diferencia de criterios diagnósticos. La prevalencia más baja puede ser justificada por el criterio estricto que fue utilizado en los pacientes del estudio. También se encontró una prevalencia dos veces más alta de adicción alcohólica en pacientes varones con esquizofrenia, en contraposición con pacientes mujeres. Este resultado es idéntico a otras investigaciones similares (Dixon 1999). Encontramos que los pacientes varones con esquizofrenia comórbida con adicción alcohólica están frecuentemente divorciados, y que son tienen una edad mucho mayor que los pacientes que sufren sólo de esquizofrenia. Las pacientes mujeres con esquizofrenia comórbida con adicción alco- 
hólica tienen un nivel de educación más bajo, no son tomadoras directas del seguro de enfermedad, a menudo son solteras, y no difieren de las pacientes mujeres con esquizofrenia sin adicción alcohólica.

Pensamos que la limitación de nuestro estudio es que hemos estudiado un amplio número de pacientes esquizofrénicos que vienen de un entorno urbano. Esto es debido a que en nuestro hospital atendemos pacientes asignados a partir de su lugar de residencia. Sin embargo, esto nos da buena información acerca del estado de los pacientes con esquizofrenia comórbida con adicción alcohólica en un entorno urbano. Además, podemos justificar las diferencias estadísticas por edad entre pacientes varones $y$ mujeres por el hecho de que los síntomas de la esquizofrenia en pacientes varones se manifiestan unos años antes, y el tratamiento de éstos también comienza antes que en las pacientes mujeres. Los pacientes hombres y mujeres con esquizofrenia comórbida con adicción alcohólica no siguen su tratamiento psicofarmacológico, de modo que tienen un número mucho más alto de hospitalizaciones y de recaídas por año. Debido a esto, su pronóstico es mucho peor, en contraste con el de aquellos pacientes con esquizofrenia sin adicción alcohólica.

La adicción alcohólica puede producir síntomas esquizofrénicos porque incrementa el nivel de monoaminas (Buljan et al. 1996, Thaller et al. 1999), especialmente el nivel de dopamina, y éste es uno de los principales causantes de la fisiopatología esquizofrénica (Pilowsky et al. 1992). La adicción alcohólica se relaciona con un incremento en la regulación de los receptores NMDA, que son (Hoffman et al. 1989) el principal origen en la neuroquímica de la esquizofrenia (Lieberman y Kooreen 1993). Por tanto, creemos que la adicción alcohólica en pacientes con esquizofrenia no es una consecuencia de un bajo nivel socioeconómico de los pacientes, como algunos autores creen (Marneros 1992), sino de las malas condiciones de vida de los pacientes que causa y/o intensifica los defectos neuroquímicos en la esquizofrenia.

La adicción alcohólica deteriora el cuadro clínico de la esquizofrenia, y también deteriora el curso y el pronóstico de la enfermedad. En las familias de los pacientes con esquizofrenia comórbida con adicción alcohólica, encontramos morbilidad psiquiátrica de sus familiares de padecer adicción alcohólica, y también de esquizofrenia comórbida con adicción alcohólica. Algunos investigadores creen que es un modelo aprendido de conducta hacia el alcohol (Keller 1990). De cualquier modo, los estudios de adopción documentan una incidencia más alta de alcohólicos entre los hijos que viven con sus padres biológicos que entre aquéllos que fueron adoptados y crecieron en familias en las que un miembro era alcohólico (Rimmer y Jacobsen 1977, Noordsy et al. 1994). Además, los análisis genéticos de las familias alcohólicas confirman la relación entre la adicción alcohólica y los genes responsables de la transmisión los receptores dopaminérgicos D2 (Noble 2000, Blum et al. 1990). Se ha probado la alteración de estos genes en la esquizofrenia (Seeman y Niznik 1990), así como en los receptores D2. Más aún, numerosos fármacos para tratar la esquizofrenia actúan sobre estos receptores (Meltzer 1999). Finalmente, podemos decir que la esquizofrenia comórbida con adicción alcohólica tiene una influencia genética, y que esta comorbilidad hace el cuadro clínico más complicado, por lo que el pronóstico de estos pacientes es bastante malo. En futuras investigaciones debemos analizar la estructura genética de los miembros de la familia de los pacientes. Y aún más, para el propósito de una psicofarmacoterapia más eficiente, 
deberíamos investigar los cambios en la neurotransmisión en pacientes con esquizofrenia comórbida con alcoholismo. De este modo, se podrían prevenir las frecuentes recaídas y prolongar la remisión de los pacientes.

\section{Bibliografía}

AMERICAN PSYCHIATRIC ASSOCIATION. Diagnostical and statistical manual of mental disorders $\left(4^{\text {th }}\right.$ edition). Washington, (DC): APA; 1994.

BATEL, P. Addiction and Schizophrenia. Eur Psychiatry 15: 115-122, 2000.

BERMAN, O.M. Severe brain dysfunction: Alcoholic Korsakoffs syndrome. Alc Healt and Res World 14: 120129, 1990.

BLEULER, E. Dementia praecox oder die gruppe der schizophrenien. Lelpzig-Wien: Deuticke; 1911.

BLUM, K., NOBLE, E.P., SHERIDAN, P.J., MONTGOMERY, A., RITCHIE, T. Allelic association of human dopamine D2 receptor gene in alcoholism. JAMA 263: 2055-2060, 1990.

BROWN, K.V., WHITE, T. Syndromes of chronic schizophrenia and some clinical correlates. Br. J. Psychiatry 161: 317-322, 1992.

BULJAN, D., BRZOVIĆ, Z., THALLER, V., BREITENFELD, D., MARUŠIĆ, S. Neurotransmitter changes in alcoholism and in the withdrawal syndrome, neurobiological tests of alcoholism. Coll. Antropol. 20: 175-182, 1996.

CANTOR, E., NORDOSTROM, L.G., McNEIL, T.H. Substance abuse in schizophrenia: a review of the literature and its correlates in Sweden. Schizophrenia Res. 48: 69-82, 2001.

CLONINGER, C.R., BOHMAN, A., SIGVARDSSON, S. Inherence of alcohol abuse: Crossfostering analysis of adopted men. Arch. Gen. Psychiatry 38: 861-868, 1981.

DIXON, L. Dual diagnosis of substance abuse in schizophrenia: prevalence and outcomes. Schizophr Res 35: 93$100,1999$.

EWING, J.A. Detecting alcoholism: The CAGE Questionnare. JAMA 252: 1905, 1984.

FENTON, W.S., McGLASHAN, T.H. Natural history of schizophrenia. Arch. Gen. Psychiatry 48: 969-976, 1991.
GUEBALY, N., HODGINS, D.C. Schizophrenia and substance abuse. prevalence issues. Can. J. Psychiatry 37: 704-710, 1992.

HAFNER, H., HEIDEN, W. Epidemiology of schizophrenia. Can. J. Psychiatry 42: 139-151, 1997.

HELZER, J.D., PRYZBOCK, T.R. The co-occurrence of alcoholism with other psychiatric disorders in the general population and its impact on treatment. J. Stud. Alcohol 49: 219-224, 1988.

HOFFMAN, P.L., RABE, C.S., MOSES, F. TABAKOFF, B. N-methyl-D-aspartate receptors and ethanol. J. Neurochem 52: 1937-1940, 1989.

KELLER, M. Models of alcoholism From days of old to nowadays. Center of Alcohol Studies Pamphlet Series. Piscataway. NY. 1990.

KING, M. A risk drinking among general practice attenders: validation of CAGE questionnare. Psychol. Med. 16: 213-219, 1986.

LOVINGER, D.M., WHITE, G., WEIGHT, F.F. Etanol inhibits NMDA-acrivated ion curent in hipocamal neurons. Science 243: 1721-1724, 1989.

MARNEROS, A. The long-term course and outcome of schizophrenia. Triangle 31: 155-162, 1992.

MELTZER, H. Treatment of schizophrenia and spectrum disorders: pharmacotherapy, psychosocial treatments, and neurotransmitter interactions. Biol. Psychiatry 46: 1321-1327, 1999.

NOBLE, E.P. Addiction and its reward process through polymorphisms of the D2 dopamine receptor gene: a review. Eur Psychiatry 15: 79-89, 2000.

NOORDSY, D.L., DRAKE, R.E., BIESANZ, J.C., McHUGO, G.J. Family history of alcholism in schizophrenia. J. Nerv. Ment. Dis. 182: 651-655, 1994.

PENICK, E.C., POWELL, B.J., NICKEL, E.J., BINGHAM, S.F., RIESENMY, K.R., READ, M.R. Comorbidity of lifetime psychiatric disorder among male alcoholics. Alcohol. Clin. Exp. Res. 18: 1289-1293, 1994.

PILOWSKY, L.S., COSTA, D.C., ELL, P.J. Clozapine, single photon emission tomography and the D2 biocade hypothesis of schiozophrenia. Lancet 340: 199-202, 1992.

REGIER, D., BURKE, J.D. Epidemiological methods in psychiatry. In Kaplan $\mathrm{Hl}$ and Sadock BJ. Comprehensive texbook of psychiatry, Williams and Wilkins, Baltimore 1995.

RIMMER, J., JACOBSEN, B. Alcoholism in schizophrenics and their relatives. J. Stud. Alc. 38: 1781-1784, 1977. 
RUBIN, E. The chemical patogenesis of alcoholinduced tissue injury. Alc Health and Research World 17: 292-298, 1993.

SEEMAN, P., NIZNIK, H.B. Dopamine receptors and transporters in parkinson disease and schizophrenia. FASEB Journal 4: 2737-2744, 1990.

THALLER, V. Alkoholizem. Alcoholism 34, Suppl. 1: 132-155, 1999.

THALLER, V., BULJAN, D., MARUŠIĆ, S. Biochemical tests in the diagnosis of alcoholism (the correlation of the sensitivity of the conventional tests for the diagnosis of alcoholism and alcohol withdraval syndrome), Eur. J. Psychiat., 13: 107-119, 1999.
THALLER, V., BREITENFELD, D., PINTARIĆ, S., IVICA, J., BULJAN, D., GOLIK-GRUBER, V., MARUŠIĆ, S., POLDRUGO, F. Self-help groups of alcoholics and war in Croatia. Alcoholism 32: 29-33, 1996.

Dirección para correspondencia:

Dalibor Karlović

University Department of Psychiatry

University hospital "Sestre milosrdnice"

Vinogradska 29

10000 Zagreb

CROATIA 